\section{AB0918 REACTIVE ARTHRITIS POST VIRAL - AN UNUSUAL PRESENTATION IN AN EPIDEMIC IN NORTH INDIA}

N. Mendiratta, R. Gupta, D. Rawal on behalf of Dr Dhiren Raval. MEdanta, MEdcity, New Delhi, India

Background: This study was performed at a Private hospital in New Delhi, India during the epidemic phase of Viral fever. The peak months of viral fever from mosquitoes (Aedes Aeygpti) like Dengue and Malaria (Anopheles)has been from July to October, till the holy festival of Diwali arrives. Chikungunya came like a wave this time along with dengue and Malaria. The worst epidemic since the last 6 years. A disease common in South India, took North India by a storm. 15-20 deaths were also reported due to Chikungunya virus. Apart from Chikungunya (serology negative) there were a lot of other viruses causing arthritis. Our study deals with post viral arthritis, a new variant which has never been described before.

Objectives: 1 . To study the pattern of arthritis after viral fever. Is it reactive or a new variant

2. To observe for the evolution of Acute viral arthritis into chronic arthritis

3. The management of Post viral arthritis

Methods: It is a retrospective study conducted at the end of the epidemic. The patients are being followed up over next 3 months to observe for resolution of symptoms, persistent arthritis or evolution into chronic form. 100 patients are being included which were examined and independently assessed by 3 different consultants.

Inclusion criteria: 1. All patients who presented with complaints of persistent joint pains and swelling preceded by fever (Average duration 4-8 weeks). 2 . Documented synovitis (Oligoarticular and polyarticular)

Exclusion criteria: 1. Known case of Rheumatoid Arthritis, Connective tissue disease, Vasculitis and Spondyloarthopathy 2. Arthalgia with no documented synovitis 3. Patients on DMARDS previously

Results: Detailed results are still under compilation as patients are under follow up (6 months) for further course.

No of patients: 100

Average Age: 47

Average Disease Duration: 6 weeks (after fever)

Average No of Joints involved: 3-4

Symmetry: All Asymmetrical (Large+ Small)

$80 \%$ patients had asymmetrical joint involvement. Most common joints were: MCP followed by PIP and then the large joints: shoulders and ankles. It was associated with significant early morning stiffness (30 minutes) like other inflammatory arthritis. $60 \%$ had response to short course of NSAIDS and low dose steroids (Injection Depomedrol $80 \mathrm{mg}$ intramuscular once a week) and recovered in 2 weeks, $30 \%$ had a prolonged course of 4-6 weeks, butdid not need any further medications. 5\% developed into Chronic arthritis (Further follow up pending)

Conclusions: Reactive arthritis is a known entity and it has typical involvement of the lower limbs, usually preceded by urinary tract infection or Gl infection. Even with Viral arthritis, the presentation of joint pains and swelling is usually during the acute fever episode. The pattern described here was different. All the patients had fever at presentation which lasted for 3-5 days and 4-6 weeks later they developed synovitis. There was characteristic involvement of Small joints of the hands (PIP and MCPS) (different from reactive). We are still in process of collecting follow up data which will give us a clue on prognosis of this arthritis and future prospects. So, what do we label it as ... Reactive arthritis-a new variant or a post viral arthritis

[1] Varache S, Narbonne V, Jousse-Joulin S, et al. Is routine viral screening useful in patients with recent-onset polyarthritis of a duration of at least 6 weeks? Results from a nationwide longitudinal prospective cohort study. Arthritis Care Res 2011:63:1565-70.

Disclosure of Interest: None declared

DOI: 10.1136/annrheumdis-2017-eular.1168

\section{AB0919 THE PROGNOSTIC FACTORS DURING OSTEOARTICULAR TUBERCULOSIS}

O. Saidane ${ }^{1}$, M. Sellami ${ }^{1}$, S. Cheikhrouhou ${ }^{2}$, I. Mahmoud $^{1}$, R. Tekaya ${ }^{1}$,

L. Abdelmoula ${ }^{1}$. ${ }^{1}$ Rheumathology; ${ }^{2}$ Parasitology, Charles Nicolle Hospital, Tunis, Tunisia

Background: Osteoarticular tuberculosis (OAT) is still common in Mediterranean countries such as Tunisia. The incidence of OAT is increasing, mainly related to expanding immunosuppressive factors and comorbidities.

Objectives: This study aimed to analyze the prognostic factors and therapeutic outcomes of OAT

Methods: Over a period of 20 years [1996-2016], patients with the diagnosis of OAT were retrospectively identified. Clinical, laboratory and radiology features in these patients were analyzed to identify the prognostic factors

Results: Sixty patients (29 men) fulfilled the diagnostic criteria, admitted to the Rheumatology Service with an average of 54 years (range: 16 to 86).We did not find any significant association between prognosis and an advanced age (>60years) at the time of diagnosis, a long diagnostic delay or initial neurologic involvement.However, we found that cervical localization in tuberculous spondylodiscitis, classified as an only infection site or in multi-level vertebral damage, was significantly associated to a poor prognosis.Our study revealed that hyperleucocytosis higher than 11500 elements $/ \mathrm{mm}^{3}$ at the initial blood count constituted the only biological bad prognostic factor during OAT. Based on radiological findings, the presence of abscess or vertebral compression fractures on bone imaging (MRI)and the presence of vertebral deformity (kyphosis or kyphoscoliosis) were strongly linked to a bad outcome.The following table (Table1) summarizes the poor prognostic factors during OAT.

Table 1. The poor prognostic factors during OAT

\begin{tabular}{lcc}
\hline PROGNOSTIC FACTOR & $\mathrm{p}$ & Odds Ratio \\
\hline White blood cell counts $>11500$ elements $/ \mathrm{mm}^{3}$ & 0,041 & 22 \\
Presence of abscess on MRI & 0,024 & 13 \\
Presence of vertebral compression fractures & 0,018 & Indefinite \\
Vertebral deformity & $<0,001$ & Indefinite \\
Cervical localization & 0,033 & 15 \\
Age $>60$ years & 0,078 & - \\
Long diagnostic delay & 0,062 & - \\
Initial neurologic damage & 0,053 & - \\
\hline
\end{tabular}

Conclusions: This study revealed that cervical localization in tuberculous spondylodiscitis, initial hyperleucocytosis, and the presence of abscess on MRI as well as vertebral compression fractures and vertebral deformity are associated with a bad outcome in OAT.

Disclosure of Interest: None declared

DOI: 10.1136/annrheumdis-2017-eular.2997

\section{AB0920 TUBERCULOSIS OSTEOMYELITIS OF THE PUBIC SYMPHYSIS: REPORT OF TWO CASES}

R. Sonia, S. Boussaid, I. Abdelkafi, S. Hela, C. Ilhem, E. Mohamed. Rheumatology, la Rabta, Tunis, Tunisia

Background: Infection of the symphysis pubis is a rare complication for less than $1 \%$ of cases of osteomyelitis. The predisposing causes reported are pelvic surgery, trauma and intravenous drug abuse. We report cases of osteomyelitis of the pubic symphysisdue to mycobacterium tuberculosis which is extremely Results: The two patients were 47 and 75 year-old women. They were admitted because of three month's history of progressive perineal pain and a hypogastric mass without fever in the first case, and bilateral inguinal pain with limited flexion and rotation of the right hip in the second one. Blood tests showed an elevated erythrocyte sedimentation rate (ESR) of $27 \mathrm{~mm}$ and $75 \mathrm{~mm}$. The leucocytes rates were at $5580 / \mathrm{mm}^{3}$ and $6000 / \mathrm{mm}^{3}$. Plain X-ray revealed irregularity and widening of the symphysis pubis. Tuberculin skin test was positivein one case and negative in the other. Chest radiograph was normal. The bacteriological cultures for tubercule bacillus in sputum and urines negative. The typhic and brucellian serological diagnosis as well as blood cultures were negative. CT scan showed irregular destruction and erosion of the pubic bone with a soft tissue mass. A biopsy of the symphysis was performed. Histologic examination of the bone material revealed a granulomatous inflammation with caseous necrosis confirming the diagnosis of tuberculosis. Anti-tuberculous treatment was prescribed and led to full recovery.

Conclusions: Tuberculosis is a major health problem in mediterranean countries, including Tunisia. These two cases present a timely reminder that tuberculosis should always be considered as part of the differential diagnosis of treatment of tuberculosis osteomyelitis of the pubic symphysis. Radiological investigations with plain X-rays, CT, MRI and bone scan are hepful. Treatment of tuberculous osteomyelitis of the pubic symphysis is based mainly on anti-tuberculous drugs.

Disclosure of Interest: None declared

DOI: 10.1136/annrheumdis-2017-eular.6940

\section{AB0921 INFECTIOUS SPONDYLODISCITIS: EPIDEMIOLOGICAL, CLINICAL, PARACLINICAL AND THERAPEUTIC ASPECTS}

A. Feki, R. Akrout, I. Sellami, M. Ezzeddine, H. Fourati, S. Baklouti. Hedi Chaker Hospital, -Sfax, Tunisia

Background: Spondylodiscitis is an infection of a disc and the two adjacent vertebrae due to the introduction of a pyogenic, usually by the haematogenous route. It's quite a rare disease accounting for $2-7 \%$ of all cases of septic osteomyelitis [1, 2].

Objectives: To study the clinical, microbiological, radiological, therapeutic and evolving of infectious spondylodiscitis

Methods: A retrospective descriptive study conducted over years in the department of rheumatology, including all patients with infectious spondylodiscitis Clinical given were collected from paper patients records.

Results: We included 67 patients. There were 38 men and 29women. The mean age was 55 years. The male to female ratio was 38:29.Risk factors of spondylodiscitis were observed in 19 patients. The approximate time from onset of symptoms to diagnosis was from 3 to 365 days (median, 132 days). Back pain was the most common symptom. Spinal syndrome was found in all patients. The most frequent location of spondylodiscitis was lumbar spine. Signs of spinal cord compression including paraplegia or paraparesis of the lower limbs were observed in 31 patients. Pachymeningitis was associated in 1 